The AstrophysicAL JournaL, 541:495-500, 2000 September 20

(c) 2000. The American Astronomical Society. All rights reserved. Printed in U.S.A.

\title{
LABORATORY ASTROPHYSICS SURVEY OF KEY X-RAY DIAGNOSTIC LINES USING A MICROCALORIMETER ON AN ELECTRON BEAM ION TRAP
}

\author{
E. Silver, H. Schnopper, S. BAndler, N. Brickhouse And S. Murray \\ Harvard-Smithsonian Center For Astrophysics, 60 Garden Street, Cambridge, MA 02138 \\ M. BARBERA \\ Osservatorio Astronomico G.S. Vaiana, Piazza del Parlamento 1, 90134 Palermo, Italy \\ E. TAKACS ${ }^{1}$ \\ Massachusetts Institute of Technology, 77 Massachusetts Avenue, 26-239, Cambridge, MA 02139-4307 \\ J. D. Gillaspy, J. V. Porto, and I. KinK \\ National Institute of Standards and Technology, 100 Bureau Drive, Gaithersburg, MD 20899 \\ J. M. LAMING \\ Naval Research Laboratory, Code 7674L, Washington, DC 20375 \\ AND \\ N. Madden, D. Landis, J. Beeman, ANd E. E. Haller \\ Lawrence Berkeley National Laboratory, 1 Cyclotron Road, Berkeley, CA 94720 \\ 2000 February 9; 2000 April 25
}

\begin{abstract}
Cosmic plasma conditions created in an electron beam ion trap (EBIT) make it possible to simulate the dependencies of key diagnostic X-ray lines on density, temperature, and excitation conditions that exist in astrophysical sources. We used a microcalorimeter for such laboratory astrophysics studies because it has a resolving power $\approx 1000$, quantum efficiency approaching $100 \%$, and a bandwidth that spans the X-ray energies from $0.2 \mathrm{keV}$ to $10 \mathrm{keV}$. Our microcalorimeter, coupled with an X-ray optic to increase the effective solid angle, provides a significant new capability for laboratory astrophysics measurements. Broadband spectra obtained from the National Institute of Standards and Technology EBIT with an energy resolution approaching that of a Bragg crystal spectrometer are presented for nitrogen, oxygen, neon, argon, and krypton in various stages of ionization. We have compared the measured line intensities to theoretical predictions for an EBIT plasma.
\end{abstract}

Subject headings: atomic data - atomic processes - line: identification - methods: laboratory plasmas - X-rays: general

\section{INTRODUCTION}

$\mathrm{X}$-ray spectroscopic measurements are used to determine the temperature distribution, density, ionization state, and elemental composition of hot cosmic plasmas. Knowledge of these basic parameters provides an understanding of physical processes in the hot universe such as atmospheric heating, transport, shock waves, and accretion. Plasmas of special interest are found in the magnetically heated coronae of late-type stars, the accretion disks surrounding collapsed stellar objects, the bubbles of gas heated by supernovae and the tunnels throughout interstellar space they create, the halos of elliptical galaxies, the nuclei of active galaxies, and the vast regions in galaxy clusters. The determination of the physical parameters that define the plasma relies on complex models of the continuum and line emissions. Experimental data are unavailable, except in rare cases, and the atomic data that are needed for these models are largely derived from theoretical work.

The current understanding of the fundamental processes occurring in highly ionized plasmas is not commensurate with the quality of data that will be obtained by the new $\mathrm{X}$-ray telescopes and spectrometers flown on $A S C A$, Chandra, and $X M M$. With a nominal goal of a $1 \%$ calibration, the Chandra X-ray Observatory, launched in 1999 July, had a 6 month ground-based calibration effort, which was followed by a comprehensive set of in-flight measure-

\footnotetext{
${ }^{1}$ Permanent address: Kossuth University, Debrecen, Hungary.
}

ments. By contrast, few individual atomic rates are known with an accuracy less than $20 \%$, and emission lines that depend on many processes (e.g., both direct excitation and cascades from recombination) are less accurately known. Dielectronic recombination rate coefficients and uncertain ionization state fractions derived from different ionization balance calculations may differ by factors of 2 or more.

To achieve the best scientific interpretation of the data from Chandra, XMM, and ASCA, theoretical calculations must be verified or modified by the results obtained from spectroscopic measurements in the laboratory. An excellent way to study the behavior of highly charged plasma ions is to confine them in an electron beam ion trap (EBIT). The EBIT produces customized, well-characterized, homogeneous plasmas well suited to a wide variety of precision measurements. The manipulation of the plasma conditions in the EBIT will generate a comprehensive database for comparison with theoretical atomic physics calculations. The EBIT is also uniquely capable of simulating specific slices through astrophysical plasma conditions (ions at rest, electrons with nearly constant energy) to allow the systematic examination of how the atomic structure and dynamics of plasma ions influence the energy release in cosmic X-ray sources. The literature contains numerous examples of EBIT applications (Gillaspy 1996; Beiersdorfer et al. 1996). Measurements of impact excitation rates, excited state lifetimes, ionization cross sections, resonant excitation and dielectronic recombination cross sections, and of course, 
precision wavelength measurements providing tests of quantum electrodynamics have all been demonstrated.

High-resolution X-ray crystal spectrometers and broadband semiconductor ionization detectors have been the traditional tools for these investigations. Although they have yielded many excellent results (Beiersdorfer et al. 1992; Brown et al. 1998), these instruments are limited either by a poor energy resolution (solid state detector) or by low efficiency caused by a narrow bandwidth (crystal spectrometer). An additional complication is the polarization dependence induced by the strong anisotropy of the EBIT electron beam (Henderson et al. 1990; Takacs et al. 1996). The effect of polarization must be included in the analysis of crystal spectrometer-derived intensities for lines excited by dielectronic electron capture and by electron impact.

In the experiments discussed here and in subsequent papers related to this work, the solid state ionization detector and the crystal spectrometer are replaced with a cryogenic X-ray microcalorimeter. The microcalorimeter combines the broadband capability of the semiconductor ionization detector with the high resolving power of a Bragg crystal. X-ray photons absorbed in the microcalorimeter are converted into heat, causing a temperature rise proportional to the X-ray energy. It is the ideal instrument for EBIT plasma studies and this was first demonstrated in a pilot experiment we carried out in 1995 (Silver et al. 1999). During the intervening time, an X-ray optic has been developed to increase the solid angle subtended by the microcalorimeter. This paper describes the first application of the microcalorimeter/optic combination to the spectroscopic study of astrophysically relevant plasma ions in an EBIT at the National Institute of Standards and Technology (NIST).

In $\S 2$ we review the operation of the EBIT. Section 3 is a description of the experimental setup and the adaptation of the microcalorimeter and X-ray optic to the EBIT. In $\S 4$ we present the X-ray spectra of highly ionized nitrogen, oxygen, neon, argon, and krypton. In $\S 5$ we compare the measured intensities of the helium-like lines to the theoretical predictions for the EBIT plasma.

\section{THE ELECTRON BEAM ION TRAP (EBIT)}

In traditional laboratory plasma $\mathrm{X}$-ray sources such as tokamaks, mirror machines, and those produced with lasers, the physical processes responsible for X-ray line formation cannot be isolated easily. The interpretation of the spectra obtained from these sources requires modeling of the charge balance together with a mix of line formation physics. In 1988 a new plasma source was developed jointly at the Lawrence Berkeley National Laboratory (LBNL) and Lawrence Livermore National Laboratory (LLNL), called the Electron Beam Ion Trap (EBIT) (Levine et al. 1988). This source operates at electron densities of $\approx 10^{12}$ $\mathrm{cm}^{-3}$. It simplifies the plasma diagnostics by eliminating the transport issues and line-of-sight integrations that make tokamak spectra difficult to interpret. The only other US EBIT facility is located at NIST (Gillaspy 1996). In an EBIT, the plasma is eliminated as the perturbing medium and is replaced by a narrow, well-defined, electron beam. At NIST, an energy-tunable, $0-150 \mathrm{~mA}$, electron beam can be accelerated to moderate energies $(700 \mathrm{eV}$ to $30 \mathrm{keV})$ by a series of cylindrical electrodes (drift tubes). The beam is focused to a small diameter $(70 \mu \mathrm{m})$ by a superconducting,
Helmholtz pair magnet that provides a maximum axial field of $3 \mathrm{~T}$. Atoms or slightly charged ions are introduced into the center of the middle drift tube, where the electron beam has its highest kinetic energy and tightest focus, are stripped of their outer electrons by impact ionization, and reach successively higher charge states. The limiting charge state is determined by the voltage applied to the center drift tube. It is reached when the ionization potential of the ion is greater than the energy of the colliding electrons. An axial trap for the ions in the center is formed by applying a small positive bias $(30-500 \mathrm{~V})$ on the end cap drift tubes located on either side of the center drift tube. Radial trapping is provided by the space charge of the electron beam itself, and also by the axial magnetic field which restricts the motion of the ions to modified cyclotron orbits. In addition to creating the highly charged ions and trapping them, the electron beam plays a third important role; it creates excited states that can decay by photon emission.

When compared with a conventional plasma that has a broader electron distribution, i.e., a Maxwell-Boltzmann distribution, the electron beam is nearly monoenergetic. It is precisely tunable and rapidly switchable in energy (at rates of several $\mathrm{keV} \mathrm{ms}^{-1}$ ). A wide range of species to be studied can be loaded into the trap either as gases or metal ions. A gas at room temperature, or a volatile liquid or solid, can be introduced as neutral atoms through a series of collimating holes through a side port. Metals can be loaded vertically (along the magnetic field lines) as slightly charged ions $(+1$ to +4$)$ using a metal vapor vacuum arc (MEVVA) ion source.

The NIST EBIT is equipped with an "event mode" data acquisition system that is used to measure transient processes and atomic lifetimes. This system is capable of switching EBIT operating parameters (beam energy, beam current, trapping voltage, etc.) at high speed and data can be time stamped to correlate it with the EBIT configuration.

\section{EBIT SPECTROSCOPY WITH A MICROCALORIMETER AND X-RAY OPTIC}

Operating at $65 \mathrm{mK}$, our microcalorimeters combine excellent energy resolution with relatively high count rate performance. The spectrometer has $95 \%$ quantum efficiency at $6 \mathrm{keV}$ and a large collecting area in the $0.2-10 \mathrm{keV}$ energy band. In the calorimeter, X-ray photons are absorbed in a foil of superconducting tin. The temperature rise is proportional to the X-ray energy and is measured with a neutron transmutation-doped (NTD) germanium thermistor that is attached to the underside of the absorber (Haller 1997). The small heat capacity of the composite calorimeter produces a relatively large temperature change ( $\approx 5 \mathrm{mK}$ ). The NTD thermistor is impedance-matched to a junction field effect transistor negative voltage feedback circuit (Silver et al. 1989). The detector used at NIST has an energy resolution of $5.9 \mathrm{eV}$ at $1.5 \mathrm{keV}$ and $7 \mathrm{eV}$ at $6 \mathrm{keV}$. The microcalorimeter eliminates the need for multiple, inefficient crystal spectrometers to measure X-ray emission lines that span the $0.2-10 \mathrm{keV}$ band. Consequently, the microcalorimeter shortens the total measurement time by several orders of magnitude. It is also insensitive to the polarization of X-ray emission.

The minimum distance from the plasma center to the vacuum wall is $110 \mathrm{~mm}$ in the NIST EBIT. The close proximity of other ongoing experiments mounted on adjacent viewing ports of the EBIT, however, made it difficult to 
locate the microcalorimeter closer than 1 meter. This would have limited the solid angle to $2 \times 10^{-7} \mathrm{sr}$, comparable to that of a crystal spectrometer, and would have made measurements extremely time consuming. A greater solid angle was obtained by viewing the EBIT plasma through an X-ray optic made from thin gold coated plastic foil (Silver, Schnopper, \& Ingram 1998; Schnopper et al. 1999). The design can satisfy a wide variety of focal lengths and energy ranges. The focused image has a half-power diameter that is $\leq 1 \mathrm{~mm}$ (Schnopper et al. 1999). For the EBIT plasma experiments, where the distance of closest approach is limited, the optic was placed equidistant $(762 \mathrm{~mm})$ from the source and detector. Since the EBIT creates a plasma in the shape of a narrow line, the X-ray optic can increase the $\mathrm{X}$-ray intensity by at least 30 times, helping to compensate for the X-ray absorption of the microcalorimeter windows at low energies (Silver et al. 1999).

\section{LABORATORY ASTROPHYSICS MEASUREMENTS}

The capability to selectively study ions of individual species is one of the major strengths of the EBIT/ microcalorimeter combination. In the real case of a cosmic plasma, temperature, and density variations exist that lead to severe line blending. An accurate diagnosis will be very difficult without having access to a database that can be established with measurements of the kind we describe here. We have surveyed the $K$ X-ray emission from various charge states of nitrogen, oxygen, and neon; $L$ and $K$ emission from argon; and $L$ emission from krypton. For nitrogen, oxygen, neon and argon, in particular, the He-like resonance $(w) 1 s^{2}{ }^{1} S-1 s 2 p{ }^{1} P$, intercombination $(x, y)$ $1 s^{21} S-1 s 2 p^{3} P$, and forbidden $(z) 1 s^{2}{ }^{1} S-1 s 2 s^{3} S$ transitions are observable. The relative strengths of these are useful as density and temperature diagnostics (Gabriel \& Jordan 1969). For the measurements presented here, we have determined the density dependent ratio, $R=Z /(X+Y)$ and the temperature dependent ratio $G=(X+Y+Z) / W$ for nitrogen, oxygen, neon, and argon and compare these values to those predicted for a model of the nonMaxwellian EBIT plasma.

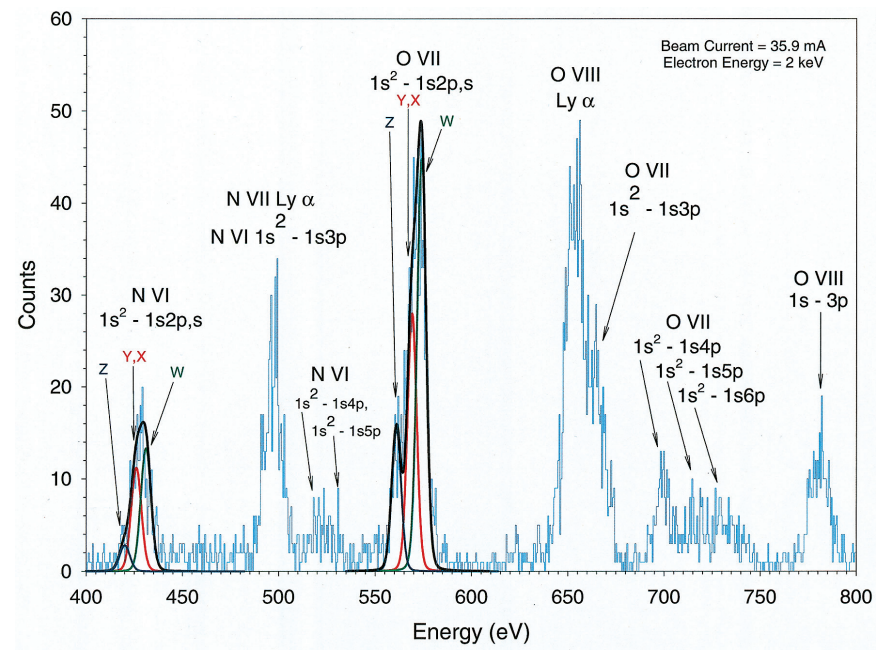

FIG. 1.-Microcalorimeter X-ray spectra from collisionally excited ions of nitrogen and oxygen from the NIST EBIT. The contributions to He-like nitrogen (N vi) and He-like oxygen (O VII) from the forbidden line $(Z$; dark blue line), intercombination line $(Y, X$; red line $)$ and resonance line $(W$; dark green line) are shown. The collection time was $\sim 30$ minutes.

\subsection{Nitrogen and Oxygen}

Oxygen and nitrogen were injected into the EBIT and for an electron beam current of $35.9 \mathrm{~mA}$ the spectrum in Figure 1 was obtained in 30 minutes. The He-like and H-like (Lyman $\alpha$ ) transitions, of nitrogen (N VI and $\mathrm{N} \mathrm{VII)}$ and oxygen (O VII and $\mathrm{O}$ VIII) are shown along with some higher transitions $\left(1 \mathrm{~s}^{2}-1 \mathrm{~s} n p ; n>2\right)$. The relative contributions of the resonance $(W$; dark green), intercombination $(X, Y$; red $)$ and forbidden ( $Z$; dark blue) lines to the He-like emission of nitrogen and oxygen are determined from a least-squares fit (solid black line) to the data (blue histogram) and for a nominal detector energy resolution of $6 \mathrm{eV}$. The values for $R$ are listed in Table 1 for both nitrogen and oxygen .

\subsection{Neon}

Neon was injected into the EBIT and X-ray spectra were obtained for various electron beam energies, electron beam currents, and trap confinement (or "dumping") times. A spectral survey of three different machine conditions is presented in Figure 2. Each spectrum was obtained in 20 minutes. A higher resolution spectrum follows in Figure 3 that shows the line structure more clearly.

The first and second panels in Figure 2 show mixtures of $\mathrm{H}$-like and He-like neon species. The data in the second panel were obtained at a higher electron beam energy, but the same electron beam current as for the data in the first panel. Since different electron beam velocities compress or stretch out the electrons along a line for the same beam
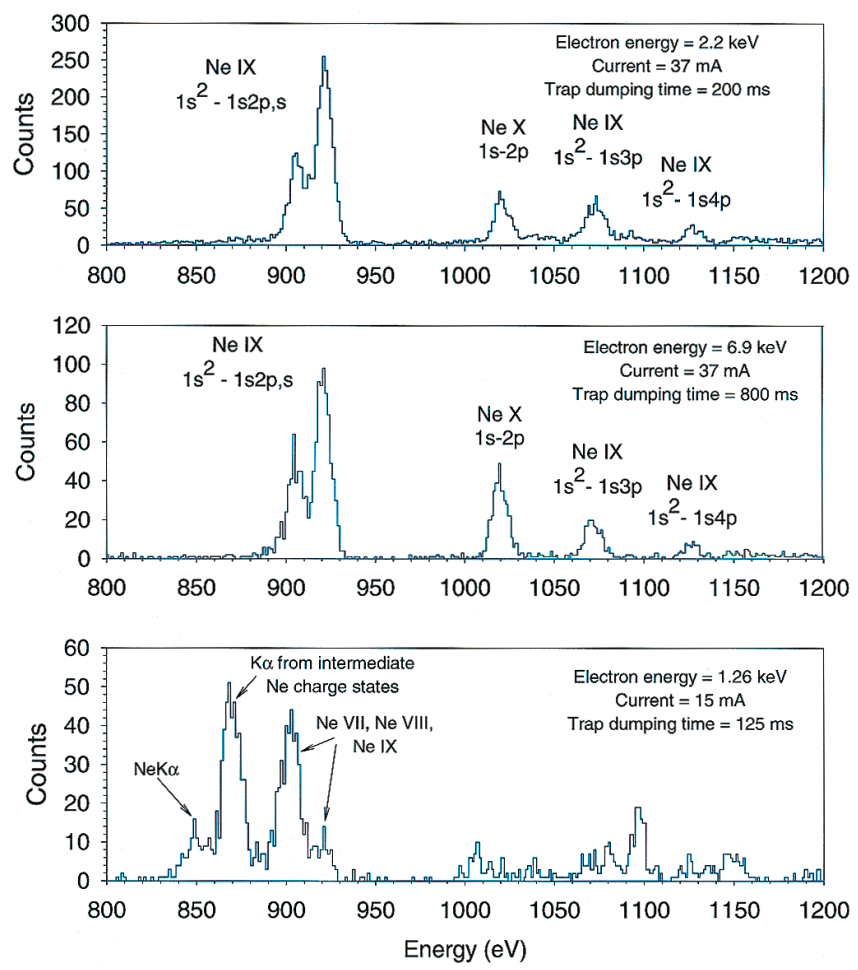

Fig. 2.-First two panels are spectra of $\mathrm{H}$ - and He-like spectra of neon ( $\mathrm{Ne} \mathrm{x}$ and $\mathrm{Ne} \mathrm{IX}$ ). The data in the second panel were obtained at the same electron beam current but higher beam energy. The intensity of the H-like $1 s \rightarrow 2 p$ line increases relative to the He-like lines. Within the He-like structure, the contribution from the intercombination line decreases at lower densities and higher beam energies (see text). The beam current and beam energy in the third panel are lower than in the second. Fewer H-and He-like ions and more ions of lower charge states (Li-like (Ne vIII) and Be-like (NevII)) are produced. The spectrum in each panel was obtained in 20 minutes. 
TABLE 1

Measured Values for $R$ AND $G$ AND INFerRed EleCtron Density

\begin{tabular}{|c|c|c|c|}
\hline Element & $\begin{array}{c}R_{\text {measured }} \\
Z /(X+Y)\end{array}$ & $\begin{array}{l}\text { Electron Density } \\
\qquad\left(\mathrm{cm}^{-3}\right)\end{array}$ & $\begin{array}{c}\mathrm{G}_{\text {measured }} \\
(X+Y+Z) / W\end{array}$ \\
\hline $\mathrm{N}$ vi......... & $0.25 \pm 0.11$ & $\begin{array}{r}+3.5 \times 10^{11} \\
4.5 \times 10^{11} \\
-1.5 \times 10^{11}\end{array}$ & $1.05 \pm 0.17$ \\
\hline O viI ........ & $0.55 \pm 0.11$ & $\begin{array}{r}+1.7 \times 10^{11} \\
6.0 \times 10^{11} \\
-1.1 \times 10^{11}\end{array}$ & $0.97 \pm 0.10$ \\
\hline Ne Ix ........ & $1.6 \pm 0.2$ & $\begin{array}{r}+4.0 \times 10^{11} \\
1.3 \times 10^{12} \\
-3.0 \times 10^{11}\end{array}$ & $0.95 \pm 0.06$ \\
\hline Ar XVII....... & $1.1 \pm 0.3$ & $2 \times 10^{12 a}$ & $0.86 \pm 0.12$ \\
\hline
\end{tabular}

NoTE.- Uncertainties shown represent one combined standard deviation

${ }^{\text {a }}$ From the machine parameters plus an estimate of the beam radius.

current, the electron density is inversely proportional to the square root of the electron beam energy. Therefore, the electron density in the second panel is approximately one half of that in the first panel. By increasing the electron beam energy and keeping the ions in the trap longer (greater trap dumping time), the probability for reaching higher charge states improves. Thus, the intensity of the $\mathrm{H}$-like ( $\mathrm{Ne} \mathrm{x}$ ) $1 s \rightarrow 2 p$ transition increases relative to the He-like transition. Within the He-like structure, the contribution from the intercombination lines decreases at lower densities and higher electron excitation energies as expected. As the density decreases, the collisional transfer to the ${ }^{3} P_{1}$ upper state from the metastable ${ }^{3} S_{1}$ level decreases. Also, the cross section for excitation from the ${ }^{3} S_{1}$ level to the ${ }^{3} P_{1}$ decreases with increasing electron energy. This is evident by the deeper valley between the peaks in the second panel. In the third panel, the electron density is the same as in the second

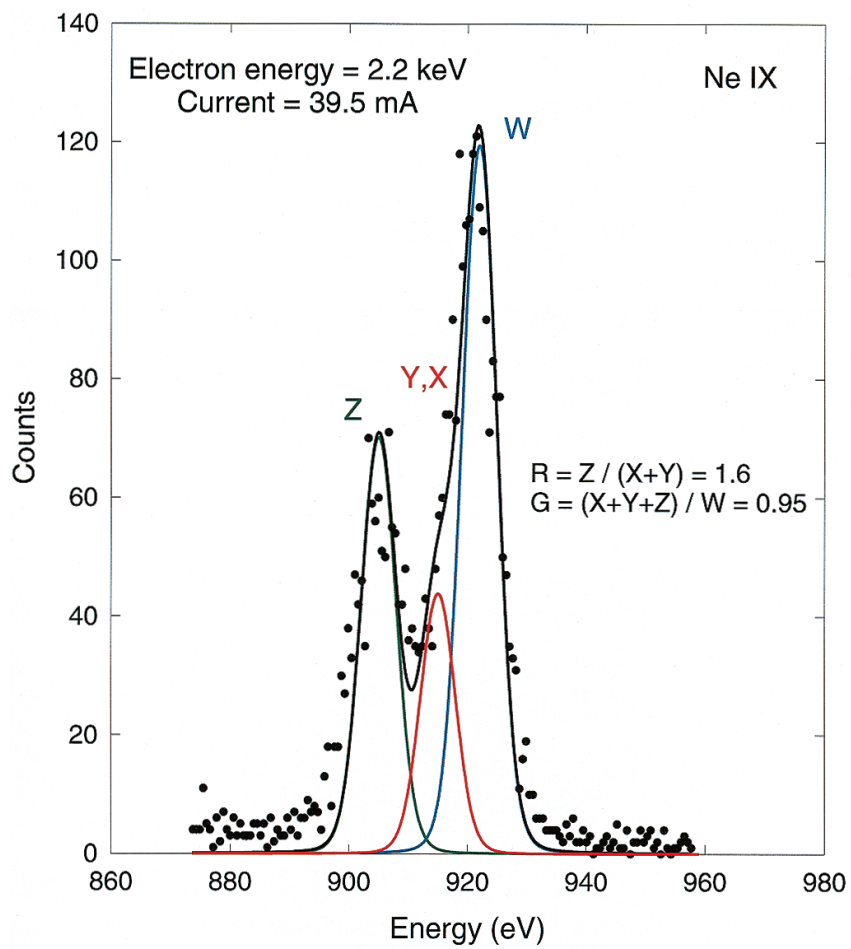

FIG. 3.-Fit to the resonance $(W$; blue line $)$, intercombination $(X, Y$; red line $)$ and forbidden $(Z$; dark green line) lines yields a line ratio $R=Z$ / $(X+Y)=1.6$ and $G=(X+Y+Z) / W=0.95$. The collection time was 29 minutes.

panel but the electron beam energy is only slightly above the He-like ionization potential $(1.12 \mathrm{keV})$. In addition, the frequency for dumping the ions from the trap is 6.4 times higher than in the previous panel. Consequently, fewer $\mathrm{H}$ and He-like ions are created. We have used the HULLAC

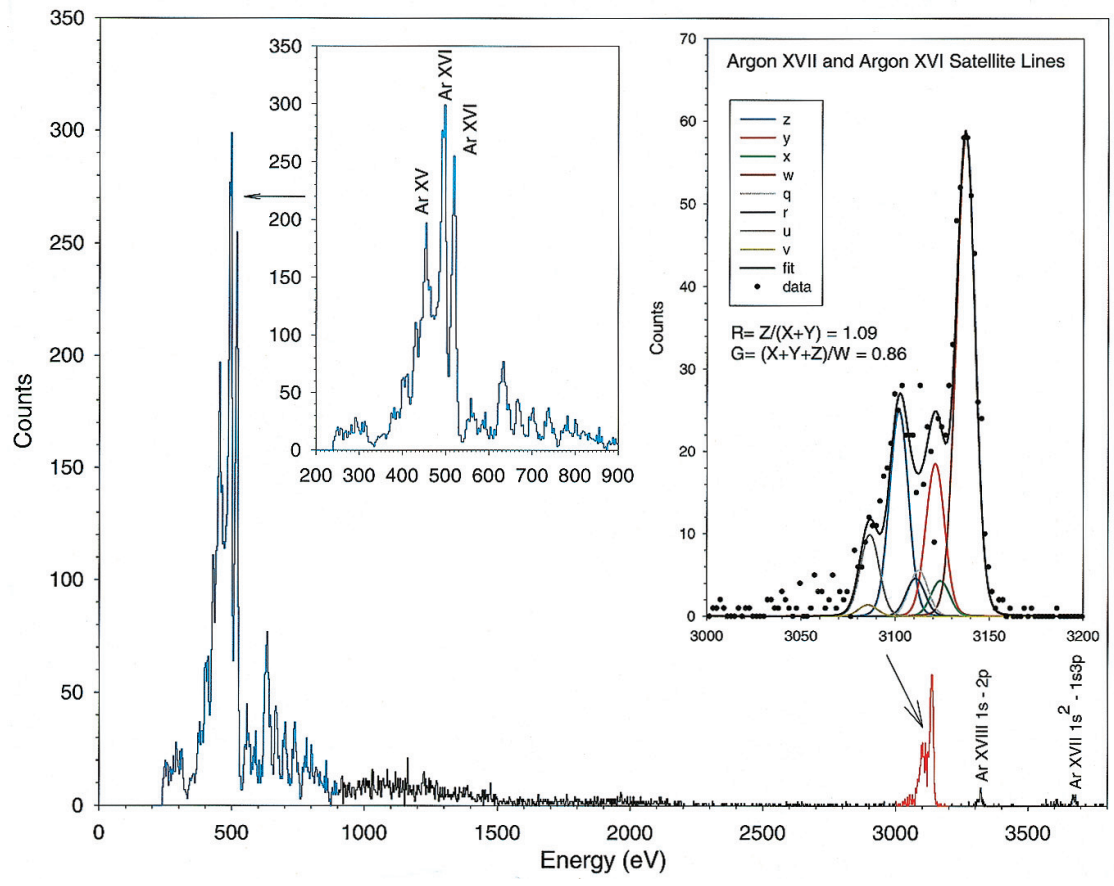

Fig. 4.- Simultaneously observed $K$ and $L$ spectra from Argon. The inset at the upper left shows the richness of the $L$ emission features from argon (blue line). The inset at the right is an expanded view of the He-like complex of Ar XVII (red line) and the additional contributions of the Ar XVI satellite lines. The collection time was 40 minutes. 


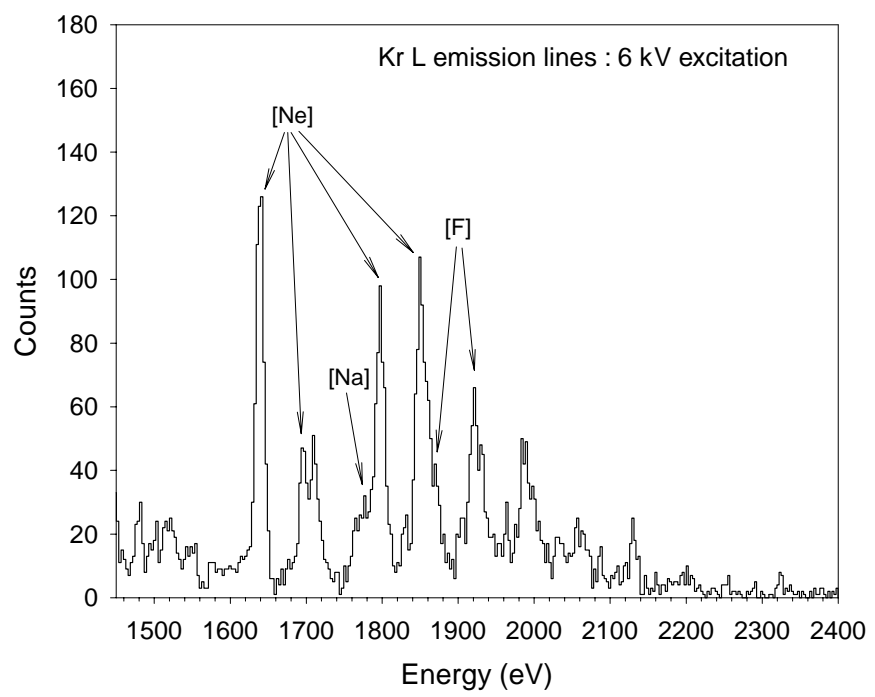

FIG. 5. $-L$ emission lines of highly charged krypton ions measured at 6 $\mathrm{keV}$ electron beam energy. Energies of the marked lines ([Ne] means Ne-like Kr, etc.) are taken from Burkhalter et al. (1979).

(Klapisch et al. 1988; Goldstein et al. 1988) suite of codes to identify the lines between 891 and $933 \mathrm{eV}$ as Ne VIII (Li-like) lines of the type $1 s 2 s 2 p, 1 s 2 s^{2}, 1 s 2 p^{2}$ to $1 s^{2} 2 s$ or $1 s^{2} 2 p$. The next peak down in energy at $\approx 870 \mathrm{eV}$ is due to similar transitions in Be-like Ne VII, and the smallest peak at $\approx 850$ $\mathrm{eV}$ is neutral neon plus any low charge state of neon that drifts into the trap from the plasma edge.

In another series of measurements the gain of the pulseheight analysis system was increased to provide higher precision in the measurement of the helium-like complex in Ne IX. The data are shown in Figure 3, where the contributions from the resonance, intercombination and forbidden lines are clearly seen. The values for $R$ and $G$ are shown and listed Table 1.

\subsection{Argon}

A broadband spectrum of argon that includes the $L$ emission lines between 400 and $700 \mathrm{eV}$ and the $K$ emission lines at approximately $3100 \mathrm{eV}$ is shown in Figure 4. Simultaneous high-resolution measurements of the $L$ and $K$ emission from the same portion of the plasma are not possible with any X-ray spectrometer other than a microcalorimeter. The argon L emission spectrum is very rich, but has received comparatively little theoretical work. Consequently a definitive identification of these lines will have to await further analysis. The argon $K$ spectrum, on the other hand, has been studied more carefully and the $K$ spectrum is fitted with the known contributions from the Ar XVII He-like lines and the satellite lines from inner shell excitation in Li-like $\mathrm{Ar}$ XVI whose transitions have the form $1 s^{2} n l-1 s 2 p n l$ with $n \geq 2$. (Phillips et al. 1993). The values for $R$ and $G$ are shown and also listed in Table 1.

\subsection{Krypton}

Krypton gas was introduced into the EBIT and X-ray spectra were obtained for different gas pressures and electron excitation energies. A sample spectrum obtained for an electron beam energy of $6 \mathrm{keV}$ is displayed in Figure 5. The lines include contributions from Li- like to $\mathrm{Mg}$-like ions and will be discussed in detail in a separate paper (Kink et al. 2000).

\section{DISCUSSION}

The broad bandwidth capability of the microcalorimeter, coupled to an EBIT with an X-ray optic, made it possible to measure simultaneously spectra from highly ionized elements of astrophysical importance. The high resolving power of the detector allowed a determination of the relative contributions to the helium-like complex from the resonance, intercombination and forbidden lines. In principle, their relative intensities provide information about the density and temperature of the non-Maxwellian EBIT plasma. These values are compared to the predictions of a model for the EBIT plasma. Levels are excited by electron impacts, and de-excited either by radiative decay or by electron impact. The energy levels, radiative decay rates and electron impact excitation cross sections are calculated using the HULLAC suite of codes. They compute bound state energy levels and wave functions using a parametric potential method, and excitation cross sections in the distorted wave approximation. Since these techniques omit resonances in excitation cross sections we were free to choose EBIT beam energies that avoid such complications and to isolate particular processes of interest.

Theoretical values for $R=Z /(X+Y)$ for $\mathrm{N}$ vi, O vII, $\mathrm{Ne}$ IX and Ar XVII are shown in Figure 6. The observed ratios for $R$ and the densities inferred for these ratios are shown by the open data points with error bars and are also given in Table 1. Nitrogen and oxygen were in the EBIT trap at the same time and the measured $R$-values for the He-like emission lines predict the same electron density for them to within the $1 \sigma$ errors. The inferred electron density of $5.8 \times 10^{11} \mathrm{~cm}^{-3}$, together with the electron beam energy of $2 \mathrm{keV}$ and beam current of $35.9 \mathrm{~mA}$, provides a measure of the electron beam radius of $69 \mu \mathrm{m}$. This is consistent with the expectation that the radius of the electron beam ( $35 \mu \mathrm{m}$ at $6 \mathrm{keV}$ ) will increase as the beam energy is reduced. (Takacs et al. 1996; Levine et al. 1988) The neon measure-

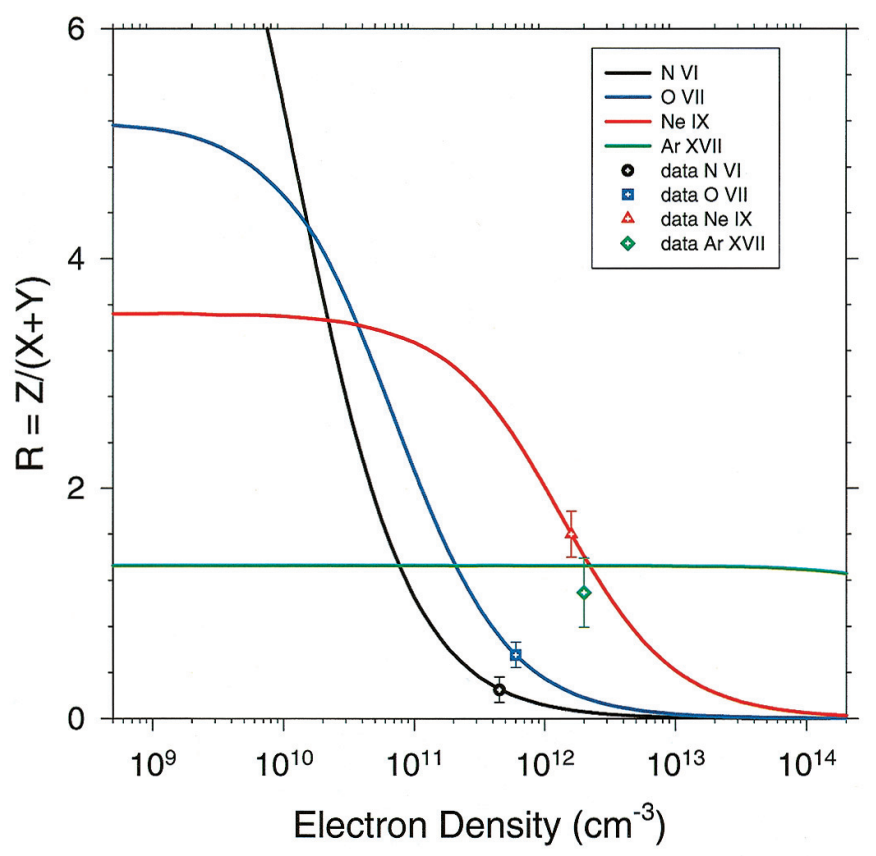

FIG. 6.-Calculated values for $R=Z /(X+Y)$ for N VI (black), $\mathrm{O}$ VII (blue line), $\mathrm{Ne} \mathrm{IX}$ (red line) and Ar XVII (green line). The measured values and their $1 \sigma$ uncertainties are shown by the open data points. 
ments were made under similar machine conditions and the $R$-values for Ne IX are consistent with those for N VI and $\mathrm{O}$ VII. These results may also be affected by the gyration of the ions in and out of the electron beam. The inferred density would be lower than the actual value. The $R$-value for the Ar XVII spectrum, obtained with a beam energy of 6.7 $\mathrm{keV}$ is consistent at the $1 \sigma$ level with density estimates of $\approx 10^{9} \mathrm{~cm}^{-3}$ to $10^{12} \mathrm{~cm}^{-3}$. However, the theory shows that the $R$-value for Ar XVII is not a sensitive indicator of the density for such high electron energies. The measured value is therefore plotted in Figure 6 at the nominal electron density that can be estimated from the beam current and beam energy.

The observed ratios for $G$ do not agree well with our theory; all experimental values indicate too much population in the $2{ }^{3} \mathrm{~S}$ and $2{ }^{3} \mathrm{P}$ states compared with $2{ }^{1} \mathrm{P}$. (The predicted $G$-value is 50 times larger than the experimental values for $\mathrm{N}$ VI, 30 times for O VII, 10 times for Ne IX, and it is 4 times larger for $\mathrm{Ar} \mathrm{XVII}$ ). This is most likely the result of charge exchange into excited states of the He-like ion from the H-like ion. The ionization balance for these observations is determined mainly by the electron impact ionization caused by the EBIT beam (whose energy is much greater than the ionization potential for the $\mathrm{H}$-like ions of $\mathrm{N}, \mathrm{O}$, and $\mathrm{Ne}$ ) and by charge exchange recombination when highly charged ions in the trap collide with the neutral atoms that provide the gas loading. Unlike radiative recombination, where the primary electron capture is in the ground state of the recombining ion, charge exchange mainly populates excited states. These subsequently decay by a radiative cascade. The relative excitation rates to the levels of the $2{ }^{3} S$ and $2{ }^{3} P$ terms are determined by the statistical weights of the levels, and, therefore, are very similar to the electron impact excitation rates for $\mathrm{N} \mathrm{VI}$, $\mathrm{O}$ VII, and Ne IX. In principle, the $R$ ratio should be essentially unchanged while the $G$ ratio will be altered. Since the $G$ ratio involves the $2{ }^{3} P$ intensity, it behaves quite differently under charge exchange than under impact excitation. The charge exchange process is not pursued further in this paper. Note, however, that the EBIT offers the potential to measure charge exchange rates into excited states. This may be important for interpreting cometary X-ray spectra or perhaps spectra from other plasmas where neutrals can coexist with highly charged ions, such as in the shock wave of supernova remnants.

\section{SUMMARY}

A microcalorimeter coupled to an EBIT with an X-ray optic provides a significant new capability for laboratory astrophysics measurements. In this pilot survey, broadband spectra obtained with an energy resolution approaching that of a Bragg crystal spectrometer have been obtained for nitrogen, oxygen, neon, argon, and krypton in various stages of ionization. The relative intensities of the He-like emission lines, in particular, were measured and compared to a theoretical model of the non-Maxwellian EBIT plasma. The electron densities that are inferred from the ratio of forbidden to intercombination lines of nitrogen, oxygen, and neon spectra agree well with theory. Charge exchange in the EBIT appears to influence the intensity of the He-like resonance line relative to the forbidden and intercombination lines. Further measurements are necessary to model properly the effects of charge exchange.

E. Silver, H. Schnopper, and S. Bandler acknowledge support in part by NASA grant NAG 5-5104, I. Kink acknowledges the Swedish Foundation for Cooperation in Research and Higher Education (STINT), and M. Laming acknowledges support from NASA contract W19539 (Applied Information Systems Research Program) and by the NRL/ONR Solar Magnetism and the Earth's Environment 6.1 Research Option. The authors wish to thank Richard Deslattes for helpful discussions and for encouraging them to make these measurements, John Raymond for suggesting measurement objectives with important implications for astrophysical spectral observations, Kate Kirby for critically reviewing the manuscript, and Frank DeFreze and Douglas J. Alderson for their excellent technical support.

\section{REFERENCES}

Beiersdorfer P., et al. 1996, Hyperfine Interactions, 99, 203

Beiersdorfer, P., Phillips, T. W., Wong, K. L., Marrs, R. E., \& Vogel, D. A. 1992, Phys. Rev. A, 46, 3812

Brown, G.V., Beiersdorfer, P., Liedahl, D .A. Widman, K., \& Kahn, S. M. 1998, ApJ, 502, 1015

Burkhalter, P. G., Shiloh, J., Fisher, A., \& Cowan, R. D. 1979, J. Appl. Phys., 50, 4532

Gabriel, A., \& Jordan, C. 1969, MNRAS, 145, 241

Gillaspy, J. D. 1996, Phys. Scr., T65, 168

Goldstein, W. H., Oreg, J., Zigler, A., Bar-Shalom, A., \& Klapisch, M. 1988, Phys. Rev. A, 38, 1797

Haller, E. E. 1997, Proc. Fourth International Symposium on Low Temperature Electronics and High Temperature Superconductors, ed. C. L. Claeys, S. I. Raider, M. J. Deen, W. D. Brown, \& R. K. Kirschman (Pennington: Electrochemical Society), 298

Henderson, J. R., et al. 1990, Phys. Rev. Lett., 65, 705
Kink, I., et al. 2000, in preparation

Klapisch, M., Bar-Shalom, A., Goldstein, W. H., Meroz, E., Chon, A., \& Cohen, M. 1988, HULLAC Code for Atomic Physics, unpublished

Levine, M. A., Marrs, R. E., Henderson, J. R., Knapp, D. A., \& Schneider, M. B. 1988, Phys. Scr., 22, 157

Phillips, K. J. H., Harra, L. K., Keenan, F. P., Zarro, D. M., \& Wilson, M. 1993, ApJ, 419, 426

Schnopper, H. W., et al. 1999, Proc. SPIE, 3766, 350

Silver, E., Schnopper, H., \& Ingram, R. 1998, US Patent Application, Serial No. 09064476

Silver, E., et al. 1999, in Proc. Eighth International Workshop on Low Temperature Detectors, ed. P. de Korte \& T. Peacock (Amsterdam: Elsevier), 2000

. 1989, Proc. SPIE, 1159, 423

Takacs, E., et al. 1996, Phys. Rev. A, 54, 1342 